\title{
Performing the nasopharyngeal and oropharyngeal swab for 2019-Novel Coronavirus (SARS-CoV-2) safely: how to dress, undress, and technical notes
}

Pasquale Di Maio, $\mathrm{MD}^{1}$

${ }^{1}$ Affiliation not available

April 30, 2020

\begin{abstract}
Background: To show how to safely perform nasopharyngeal and / or oropharyngeal swabs for 2019-Novel Coronavirus.

Methods: The video describes in detail the dressing and undressing procedures of health personnel, with the appropriate personal protective equipment. Technical notes for the execution of the nasopharyngeal and oropharyngeal swab are also provided, to avoid sampling errors.

Results: The undressing phase is the procedure with the highest risk of self-contamination for the health worker. Following the various steps as shown in the video, there were no cases of contagion among the otolaryngology team appointed to perform the swabs for SARS-CoV-2 testing.

Conclusions: This study demonstrates the technical feasibility of safely performing nasopharyngeal and/or oropharyngeal swabs for identification of SARS-CoV-2 viral RNA.
\end{abstract}

\section{Authors}

Pasquale Di Maio, M.D., ${ }^{1}$ Oreste Iocca, M.D., D.D.S., ${ }^{2}$ Antonio Cavallero M.D., ${ }^{1}$ Marco Giudice M.D.

${ }^{1}$ Giovanni Borea Civil Hospital, Department of Otolaryngology-Head and Neck Surgery, Sanremo, Italy

${ }^{2}$ Humanitas Clinical and Research Center - IRCCS -, via Manzoni 56, 20089 Rozzano (MI)

Corresponding author: Pasquale Di Maio, M.D. Giovanni Borea Civil Hospital, Via Giovanni Borea, 56, 18038, Sanremo (IM), Italy +39 3930200889 email: dimaio.p86@aliceposta.it

\section{Acknowledgments}

The authors would like to thank Dr. Giuseppe Garo, Dr. Cristina Ghirardi and Dr. Carlotta Tola for participating in the video recording. In addition, we are grateful to Ms. Rachel A. Elliott and Ms. Cristina Marconi for their support in editing the manuscript and video.

\section{INTRODUCTION}

Since its first appearance in Wuhan, China, in December 2019, the SARS-CoV-2 virus responsible for coronavirus disease 2019 (COVID-19) has progressively spread around the world. ${ }^{1}$ And to date (April 15, 2020), according to the Johns Hopkins Coronavirus Resource Center, the outbreak involved 185 countries worldwide, with 128,011 people dead. ${ }^{2}$ Healthcare workers were among the hardest hit by this infection. This was evident from reports, both from China and Italy, which revealed infection rates between medical staff between 3.8 and $20 \%{ }^{1}$ 
The tests currently used for the direct identification of SARS-CoV-2 are mainly aimed at detecting viral RNA through nucleic acid amplification, generally using the polymerase chain reaction (PCR). The nasopharynx and/or oropharynx are the most tested sampling sites using swabs, with a slightly greater sensitivity shown by the nasopharyngeal swab. ${ }^{3}$ This video shows the step-by-step procedure for properly dressing and undressing, demonstrates how to wear the personal protective equipment (PPE), and provides some technical notes on the execution of the nasopharyngeal and oropharyngeal swab for COVID-19. All of this serves to avoid the risk of contagion of the health worker. The video is based on our experience at the Giovanni Borea Civic Hospital in Sanremo, where in the last month, strictly following the CDC and WHO guidelines, ${ }^{4,5}$ the otolaryngology team has performed about 900 nasopharyngeal (95\%) and oropharyngeal (5\%) swabs. Following these steps, none of the operators have been infected as of the present date.

Rich media available at https://youtu.be/JhJu7_OBVTw

\section{VIDEO DESCRIPTION}

\section{Dressing phase}

The dressing phase takes place in a changing room, close to the entrance of the COVID area but separate from it. It requires the execution of the following steps: 1) remove all jewelry and personal items; 2) practice good hand hygiene with soap and water or alcohol solution; 3) put on a first pair of gloves; 4) put on the disposable full body scrubs; 5) put on the cap 6) wear a Filtering Facepiece Particles mask, FFP2 or FFP3 (possibly covered with a surgical mask); 7) wear protective goggles and/or a protective visor; 8) wear the shoe covers; 9) put on the second pair of gloves (which must cover the wrist of the full body scrubs). Figure 1 shows the healthcare professional at the end of the dressing phase.

\section{Nasopharyngeal swab}

The patient wears a surgical mask. She is informed about the procedure that is about to be performed. The patient must be seated in a comfortable position with his head resting on the back of the chair. The patient's head must be placed on a horizontal plane parallel to the floor. The patient is asked to lower the mask by uncovering only her nose and keeping the mouth closed. After opening the swab in a sterile manner, the operator positions the patient laterally in order to avoid direct droplet from sneezing or coughing. The swab is inserted gently into the nostril. The insertion must be parallel to the floor of the nasal fossa. The swab is kept medial and facing the nasal septum (do not insert the swab upwards, as in this case the swab stops at the level of the nasal turbinates and the viral RNA sampling may not be significant). It is important to use the mark on the swab stick as a depth reference (Figure 2). However, when the operator feels an obstacle to further introduction, it means that the swab will have reached the posterior wall of the nasopharynx. With clockwise and counterclockwise movement, repeatedly rub the swab against the posterior wall of the nasopharynx (Figure 3), for about 10-15 seconds. Extract the swab from the nostril taking care not to contaminate it upon exit, introduce it into the test tube and break it at the mark on the stick. In case of difficulty in introducing the swab into a nasal fossa due to deviation of the nasal septum or hypertrophy of the inferior turbinates, proceed with the same technique in the contralateral nasal fossa. The endoscopic vision shown in the video has a didactic purpose only, the nasopharyngeal swab should not be performed with the aid of the endoscope.

\section{Oropharyngeal swab}

If it is impossible to perform the nasopharyngeal swab (for unfavorable anatomical nasal conditions or because it is not tolerated by the patient), the oropharyngeal swab can be performed as a second choice. The patient is asked to lower the mask by exposing the mouth and to fully open it. The swab is inserted into the oral cavity until it reaches the level of the oropharynx. The sampling is carried out on the uvula, the palatine tonsils and/or the posterior wall of the oropharynx. The contact with the tongue and other areas of the oral cavity should be carefully avoided.

\section{Undressing phase}


We recommend that the undressing phase takes place in a dedicated changing room, located immediately upon leaving the COVID area. It involves the following steps: 1] remove the second pair of gloves and replace it with a pair of clean gloves; 2] remove the disposable full body scrubs and dispose it in the appropriate container (the front and sleeves of the dress are contaminated, so open the zipper or untie the laces, move it away from the neck and shoulders, touching only inside the garment, turn the lab coat upside down, fold or roll it up and throw it away); 3] remove the first pair of gloves (the outside of the gloves is potentially contaminated, therefore first remove one glove by grasping and pulling it from the outside, then remove the other glove by inserting a finger into the cuff of the glove and thus remove it, taking care not to touch its external part); 4] remove the glasses and/or the protective visor (their front is potentially contaminated, therefore to remove them, grasping the side sections, and place them in a bag for disinfection); 5] remove the mask: first the surgical mask, and then the FFP2 or FFP3, (the front of the mask is potentially contaminated, therefore after having loosened the rear laces, grasp the front of the mask, move it away from the face and remove it); 6] remove cap and shoes covers if not already removed upon removal of the full body scrubs; 7] remove the second pair of gloves; 8] practice good hand hygiene with soap and water or alcohol. For technical reasons, the undressing phase in the video takes place in the same area where the dressing took place, but we remark the importance of undressing in a separate, dedicated area. Figure 4 shows the path that the operator should follow to avoid contamination of the rooms, starting from the dressing phase until returning to the clean common area.

\section{CONCLUSION}

This report details the indications that should be followed by the healthcare professional during the execution of the nasopharyngeal and/or oropharyngeal swab for COVID 19. Particular attention must be paid during the undressing phase, due to the high risk of contagion if this phase is not correctly performed. We hope that this manuscript and related video can serve as a guide to safely perform high airway sampling for COVID 19.

\section{REFERENCES}

1. Kowalski LP, Sanabria A, Ridge JA, et al. COVID-19 Pandemic: Effects and Evidence-Based Recommendations for Otolaryngology and Head and Neck Surgery Practice. Head Neck . 2020 Apr 9. doi: 10.1002/hed.26164. Online ahead of print.

2. Johns Hopkins Coronavirus Resource Center. https://coronavirus.jhu.edu/map.html.

3. Patel R, Babady E, Theel ES, et al. Report From the American Society for Microbiology COVID-19 International Summit, 23 March 2020: Value of Diagnostic Testing for SARS-CoV-2/COVID-19. mBio . 2020;11(2):00722-20.

4. World Health Organization. Rational Use of Personal Protective Equipment for Coronavirus Disease 2019 (COVID-19).; 2020. https://apps.who.int/iris/bitstream/handle/10665/331215/WHO-2019nCov-IPCPPE_use-2020.1-eng.pdf.

5. CDC PPE Sequence. Centers for Disease Control and Prevention. https://www.cdc.gov/hai/pdfs/ppe/ppesequence.pdf.

\section{Figure legends}

Figure 1: The health worker with a full body gown and all PPE for the safe execution of nasopharyngeal and/or oropharyngeal swabs.

Figure 2: The mark on the swab stick indicates the correct insertion depth.

Figure 3: Endoscopic view of the swab on the posterior wall of the nasopharynx.

Figure 4: The path that the healthcare professional should follow to avoid contaminating the rooms. A) Changing room for dressing; B) COVID area for the execution of the swabs; C) Changing room for undressing; D) Clean external common area 


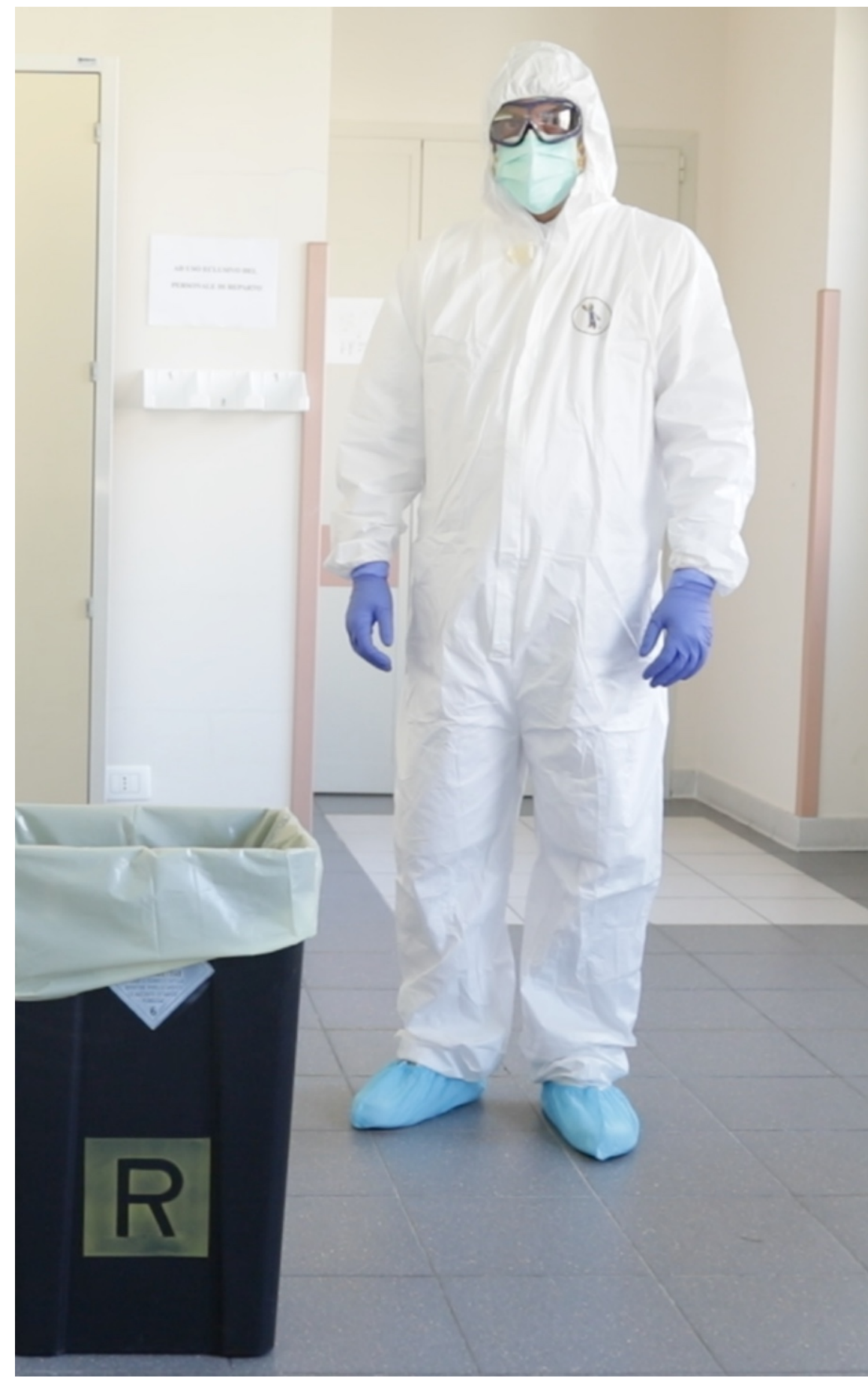



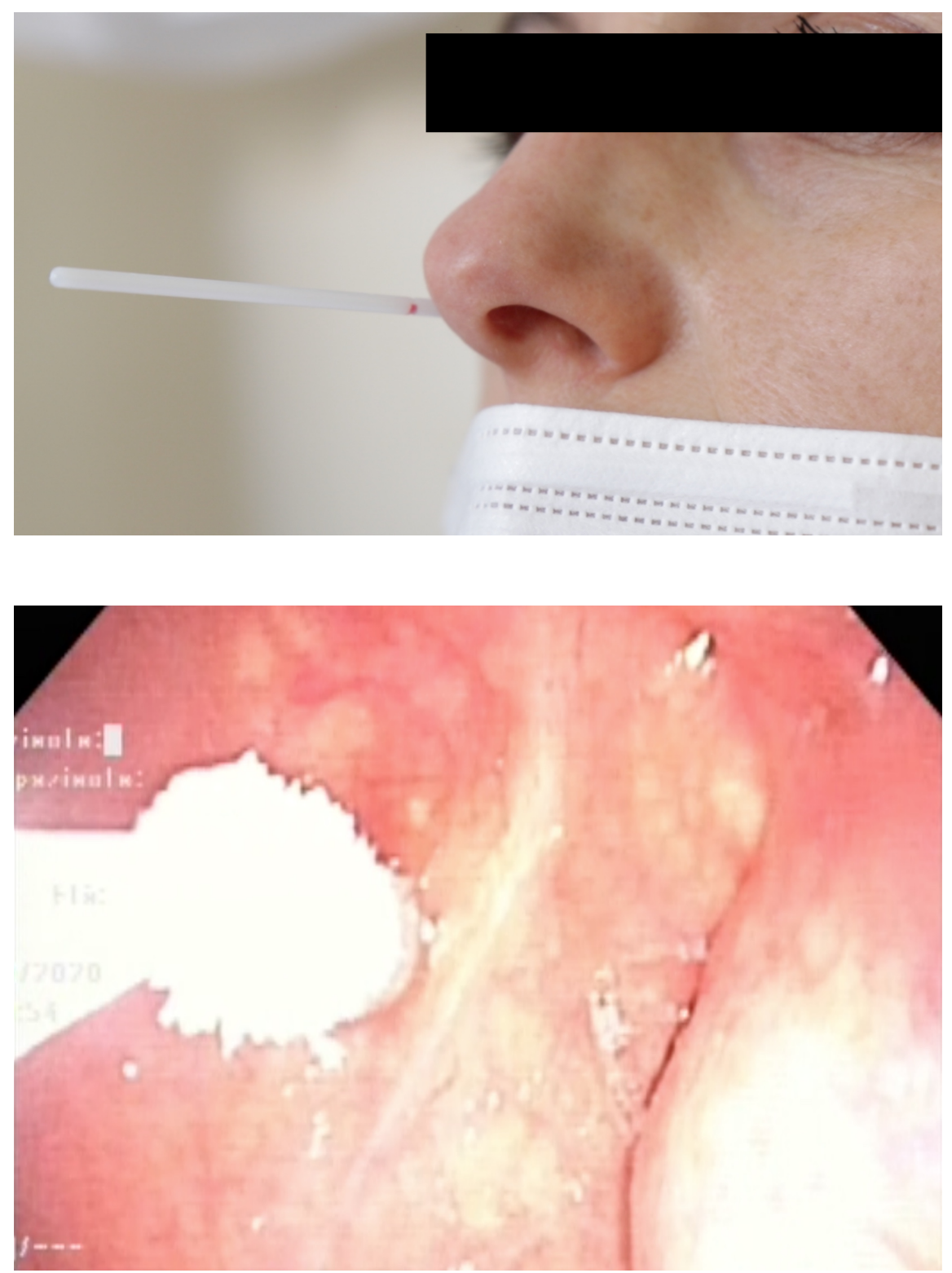


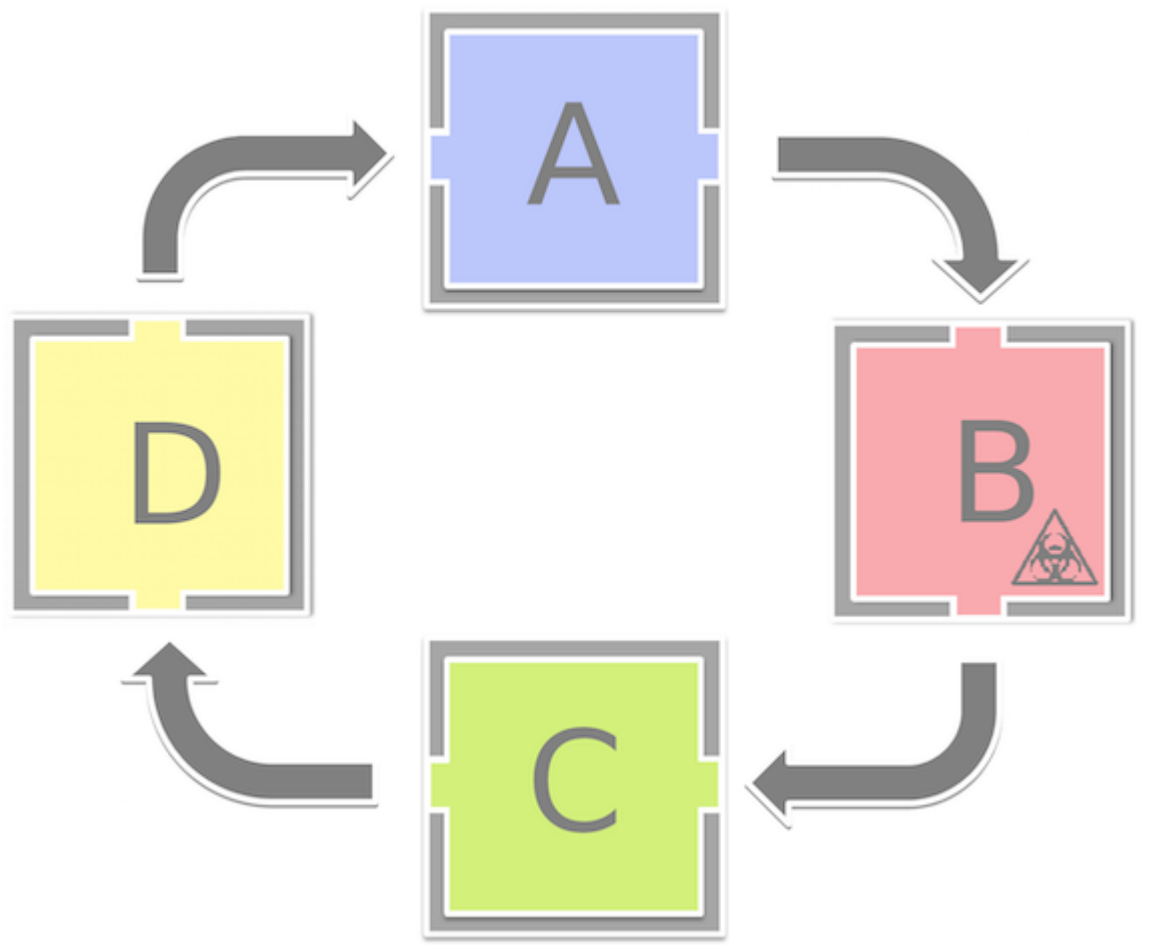

